\title{
Photopolymerization of Methyl Methacrylate with Oligo(methyl methacrylate) Containing 1-Benzyl-1,4-dihydropyridine Residue
}

\author{
Kenzo TABUCHI and Naokazu SAKOTA* \\ Department of Industrial Chemistry, Niihama Technical College, \\ 7-1 Yagumo-cho, Niihama 792, Japan \\ *Sakota Chemical Development and Research Institute Co., Ltd., \\ 402 Nakajima, Sumiyoshi-cho, Higashinada-ku, Kobe 658, Japan
}

(Received October 5, 1983)

\begin{abstract}
KEY WORDS Photopolymerization / Methyl Methacrylate / Oligo(methyl methacrylate) / Dihydropyridine / 1-Benzyl-1,4-dihydronicotinamide /
\end{abstract}

In the previous paper, ${ }^{1}$ we reported that the photopolymerization of methyl methacrylate (MMA) with $N$-benzylpyridinium bromide (BPB) in dichloromethane gave an oligo(methyl methacrylate) (OMMA) containing a 1-benzyl-1,4-dihydropyridine residue. Recently, we found that OMMA was capable of photoinitiating the polymerization of MMA. Dihydropyridines are of interest as models for NADH (nicotinamide adenine dinuclotide) coenzymes. ${ }^{2,3}$ The nonenzymatic reduction of various substrates with 1,4-dihydropyridines has been studied extensively. Only a few reports has appeared on polymerization with NADH models, ${ }^{4}$ and should be of interest whether the 1,4-dihydropyridine residue is involved in the polymerization with OMMA.

MMA, BPB and dichloromethane were placed in a hard glass ampoule, which was then degassed, filled with nitrogen, and sealed under vacuum. After irradiation by a Toshiba Fluorescent Chemical Lamp FL20SBL at $303 \pm 0.5 \mathrm{~K}$ in the same apparatus used previously, the contents were evaporated in vacuo to dryness. The conversions determined by gravimetry are plotted against irradiation time in Figure 1. The polymerization started im- mediately following irradiation, but soon ceased at about $0.8 \%$ conversion. With prolonged irradiation beyond $1.5 \mathrm{~h}$, however, the polymerization started again with greater speed than initially and the conversion increased in proportion to the irradiation time. Although the polymerization within one hour was known to be a dead-end polymerization giving OMMA, ${ }^{1}$ the subsequent photopolymerization seemed to proceed by some other

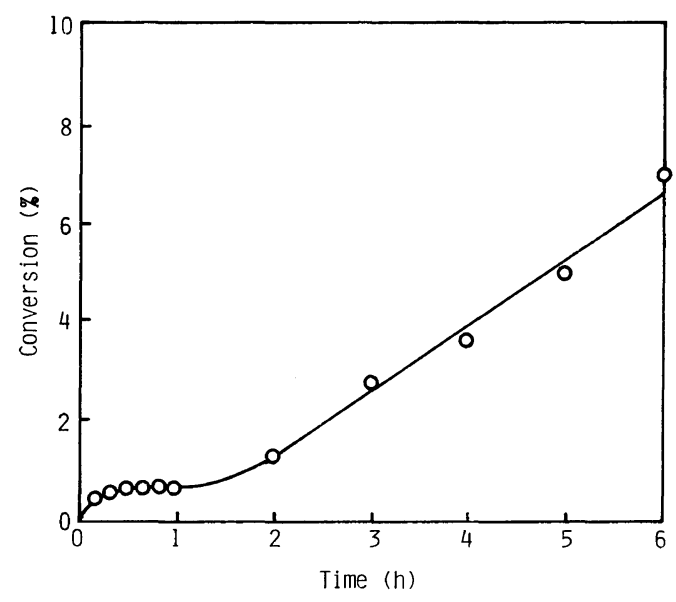

Figure 1. Photopolymerization of MMA with $\mathrm{BPB}$ in dichloromethane: MMA, $5 \mathrm{~cm}^{3} ; \mathrm{CH}_{2} \mathrm{Cl}_{2}, 5 \mathrm{~cm}^{3} ; \mathrm{BPB}$, $1 \times 10^{-3} \mathrm{~mol} \mathrm{dm}^{-3}$; temp, $303 \mathrm{~K}$. 


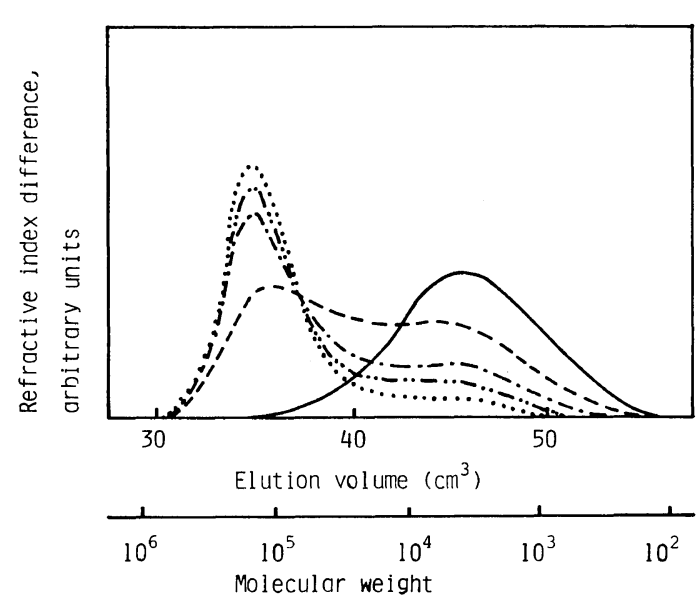

Figure 2. Gel permeation chromatograms for PMMA obtained by photopolymerization of MMA with BPB in dichloromethane under the conditions indicated in Figure 1: polymerization time (h), 1 (-), $2(----), 3$ $(--), 4$ (---) 5 (-----). GPC measurement: column, Shodex A802-A803-A805; eluent, THF.

mechanism, presumably involving initiation with OMMA. This was supported by the results of molecular weight distribution measurement. The molecular weight distributions of the OMMA were measured by gel permeation chromatography, and the refractive index difference was plotted against elution volume, as shown in Figure 2. Distinct differences in the elution patterns could be observed in the two polymerization stages. After irradiation for $1.5 \mathrm{~h}$, the polymer showed a broader molecular weight distribution than that during the initial stage and that obtained after a 5-h period of irradiation had a relatively high molecular weight of about 100,000 . To clarify the contribution of the terminal bromo group to the photoinitiation, OMMA was synthesized by the photopolymerization of MMA with $N$-benzylpyridinium thiocyanate (BPT) as described in the preceding paper. ${ }^{5} \mathrm{~N}$ Ethylpyridinium bromide (EPB) was also used instead of BPB for the photopolymerization of MMA. The features of conversion-time relation and molecular weight distributions in these experiments were nearly the same as those in Figures 1 and 2. This suggests the
Table I. A large scale photooligomerization of MMA with $\mathrm{BPB}^{\mathrm{a}}$

\begin{tabular}{|c|c|c|c|}
\hline $\begin{array}{l}\text { Irradiation } \\
\quad \text { time }\end{array}$ & $\begin{array}{c}\text { Number of } \\
\text { BPB- }\end{array}$ & Conversion & $M$ \\
\hline $\mathrm{h}$ & addition & $\%$ & \\
\hline 0.5 & $1^{b}$ & 0.99 & 950 \\
\hline 1.0 & 2 & 2.13 & 1,000 \\
\hline 1.5 & 3 & 3.23 & 1,000 \\
\hline 2.0 & 4 & 4.53 & 1,000 \\
\hline
\end{tabular}

a MMA, $15 \mathrm{~cm}^{3} ; \mathrm{CH}_{2} \mathrm{Cl}_{2}, 30 \mathrm{~cm}^{3}$; the $\mathrm{BPB}$ solution in $\mathrm{CH}_{2} \mathrm{Cl}_{2}\left(1 \times 10^{-2} \mathrm{moldm}{ }^{-3}\right), 5 \mathrm{~cm}^{3}$, added at 30 minute intervals; temp, $303 \mathrm{~K}$.

b Before irradiation a $5 \mathrm{~cm}^{3}$ portion of the BPB solution was added to the polymerization system.

involvement of the 1,4-dihydropyridine residue in the photopolymerization of MMA with OMMA.

To confirm the participation of the 1,4dihydropyridine residue in the polymerization, a large quantity of OMMA was prepared by the following procedure. MMA, BPB and dichloromethane were charged into the reaction vessel described in the preceding paper, ${ }^{5}$ and irradiated at $303 \mathrm{~K}$ under bubbling nitrogen, using a Fuji Glass High Pressure Mercury Lamp HL-100 (100 W). Additional BPB was charged every $30 \mathrm{~min}$ from a dropping funnel. After irradiation, the contents were evaporated in vacuo to dryness. The conversion was determined by gravimetry and the molecular weight was estimated by GPC. The results are shown in Table I and Figure 3. The molecular weight of the polymer obtained was constant at about 1,000 independent of the irradiation time. The conversions are represented by open circles and the conversiontime relation curves expected from the results in Figure 1 are indicated by dotted lines. These results imply that the photopolymerization of MMA is a dead-end polymerization. The OMMA obtained was collected and purified by chromatography on a silica gel column with acetone. Similar procedures were applied to OMMA prepared with BPT and EPB. The 


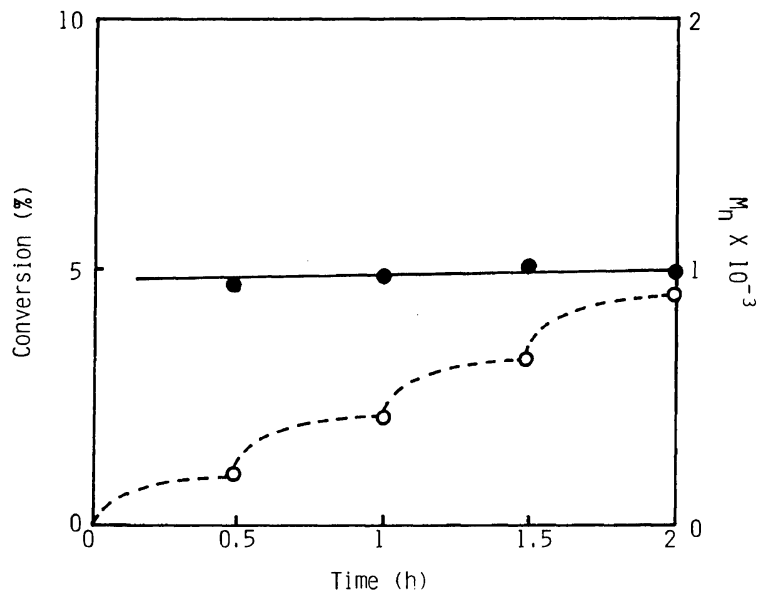

Figure 3. A large scale photooligomerization of MMA with BPB: $\mathrm{MMA}, 15 \mathrm{~cm}^{3} ; \mathrm{CH}_{2} \mathrm{Cl}_{2}, 30 \mathrm{~cm}^{3}$; the BPB solution in $\mathrm{CH}_{2} \mathrm{Cl}_{2}\left(1 \times 10^{-2} \mathrm{moldm}^{-3}\right), 5 \mathrm{~cm}^{3}$, added at $30 \mathrm{~min}$ interval; temp, $303 \mathrm{~K} ; \bigcirc$, conversion; $\mathbf{O}$, molecular weight.

Table II. Molecular weight and elemental analysis data of the purified OMMA

\begin{tabular}{|c|c|c|c|c|c|c|c|c|c|}
\hline \multirow{3}{*}{ Oligomer $^{a}$} & \multirow{3}{*}{$M_{n}^{\mathrm{b}}$} & \multicolumn{8}{|c|}{ Elemental analysis $/ \%$} \\
\hline & & \multicolumn{4}{|c|}{ Calcd $^{\mathrm{d}}$} & \multicolumn{4}{|c|}{ Found } \\
\hline & & $\mathrm{Br}$ & $\mathrm{C}$ & $\mathrm{H}$ & $\mathrm{N}$ & $\mathrm{Br}^{\mathrm{e}}$ & $\mathrm{C}$ & $\mathrm{H}$ & $\mathrm{N}$ \\
\hline OMMA (BPB) & 820 & 9.76 & 59.27 & 7.02 & 1.71 & 8.60 & 58.61 & 7.03 & 1.09 \\
\hline OMMA (BPT) & 650 & - & 62.95 & 7.04 & 4.31 & - & 58.53 & 6.95 & 3.43 \\
\hline OMMA (EPB) & 800 & 10.00 & 56.40 & 7.37 & 1.75 & 10.65 & 55.66 & 7.26 & 1.01 \\
\hline
\end{tabular}

${ }^{a}$ In parentheses are shown the pyridine derivatived with which the oligomers were obtained.

b By GPC.

- Using a Yanagimoto CHN Coder MT-3.

d Calculated from the molecular weights $\left(M_{n}\right)$ and structures $1 \mathrm{a}, 1 \mathrm{~b}$ and $1 \mathrm{c}$, respectively.

e By Konishi and Ishizuka's method. ${ }^{6}$

molecular weight and elemental analysis data of the purified OMMA are given in Table II. The elemental analysis data suggest the following structure for OMMA.

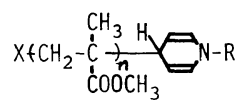

$$
\begin{aligned}
& \text { 1a }: X=\mathrm{Br}, \mathrm{R}=\mathrm{PhCH}_{2} \\
& \text { 1b }: X=\mathrm{SCN}, \mathrm{R}=\mathrm{PhCH}_{2} \\
& \text { 1c }: X=\mathrm{Br}, \mathrm{R}=\mathrm{CH}_{3} \mathrm{CH}_{2}
\end{aligned}
$$

The excitation and fluorescence spectra of OMMA (BPB) measured in dichloromethane resembled those of 1-benzyl-1,4-dihydronicotinamide (BNAH). Therefore, one of the end groups of the oligomers may possibly be the 1,4-dihydropyridine residue.

To determine whether the contribution to the photoinitiation was from either the 1benzyl-1,4-dihydropyridine residue or bromo group, the photopolymerization of MMA was carried out with BNAH and methyl $\beta$-bromo$\alpha$-methylpropionate (MBMP) as models for 
the end-groups of OMMA. The former photoinitiated the polymerization of MMA, but not the latter. The photopolymerization of MMA with BNAH was accelerated by the addition of MBMP. The photopolymerization of MMA with BNAH apparently proceeded by a radical mechanism, since the polymerization was inhibited by 1,1-diphenyl-2-picrylhydrazyl and the copolymer composition curve obtained for the photo-copolymerization of styrene and MMA with BNAH corresponded to that of the radical polymerization.

BNAH has often been used as a reductant for various substrates, ${ }^{7-9}$ but the exact mechanism is still not completely understood. For example, BNAH has been reported to reduce dimethyl maleate and fumalate to dimethyl succinate with photoactivation at room temperature. ${ }^{7}$ We found that the fluorescence from $\mathrm{BNAH}^{*}$ (the excited state of BNAH) was eliminated by MMA. Accordingly, BNAH* presumably reacts with MMA to form a radical, which then initiates the polymerization of MMA. But acceleration by MBMP in the present study may possibly be due to the reaction between alkyl halides and BNAH,${ }^{9}$ in which one-electron transfer from BNAH* to alkyl halides has been proposed as a reaction mechanism.
It is concluded that the OMMA obtained by the photooligomerization of MMA with BPB in dichloromethane photoinitiates the polymerization of MMA, and that the photoinitiation can be attributed to the 1-benzyl-1,4dihydropyridine residue contained as an endgroup of OMMA and enhanced by the bromo group bonded to the other end-group.

Acknowledgment. The authors wish to thank Professor Kiichi Takemoto of Osaka University for his kind encouragement.

\section{REFERENCES}

1. K. Tabuchi and N. Sakota, Polym. J., 15, 57 (1983).

2. U. Eisner and J. Kuthan, Chem. Rev., 72, 1 (1972), and references cited therein.

3. D. M. Stout and A. I. Meyer, Chem. Rev., 82, 223 (1982), and references cited therein.

4. S. Shinkai, T. Tsuno, Y. Asatani, and O. Manabe, Chem. Lett., 1439 (1982).

5. K. Tabuchi and N. Sakota, Polym. J., 15, 713 (1983).

6. H. Konishi and T. Ishizuka, Kobunshi Kagaku, 17, 169 (1960).

7. Y. Onishi, M. Kagami, and A. Ohno, Chem. Lett., 125 (1975).

8. N. Ohno, R. Tamura, and A. Kaji, J. Am. Chem. Soc., 102, 2851 (1980).

9. S. Fukuzumi, K. Hironaka, and T. Tanaka, Chem. Lett., 1583 (1982). 